\title{
X-Ray Diffraction Analysis of Nano Crystalline Ceramic $\mathrm{PbBaTiO}_{3}$
}

\author{
V. S. Vinila', Reenu Jacob ${ }^{2}$, Anusha Mony ${ }^{1}$, Harikrishnan G. Nair ${ }^{1}$, Sheelakumari Issac ${ }^{3}$, \\ Sam Rajan ${ }^{2}$, Anitha S. Nair ${ }^{4}$, D. J. Satheesh' ${ }^{1}$, Jayakumari Isac ${ }^{{ }^{*}}$ \\ ${ }^{1}$ Centre for Condensed Matter, Department of Physics, CMS College, Kottayam, India \\ ${ }^{2}$ Department of Physics, CMS College, Kottayam, India \\ ${ }^{3}$ Department of Chemistry, UC College, Aluva, India \\ ${ }^{4}$ Department of Physics, D. B. College, Parumala, India \\ Email: ${ }^{*}$ drjayacmscollege@gmail.com
}

Received 24 July 2014; revised 23 August 2014; accepted 15 September 2014

Copyright (C) 2014 by authors and Scientific Research Publishing Inc.

This work is licensed under the Creative Commons Attribution International License (CC BY).

http://creativecommons.org/licenses/by/4.0/

(c) (7) Open Access

\begin{abstract}
X-ray crystallography is concerned with discovering and describing the crystal structure. Hightemperature superconductivity in ceramic oxides is a new technology in which advances are occurring at a rapid pace. Here, the author describes some properties of a new nano crystalline ceramic type II superconductor, PbBaTio. Type II superconductors are usually made of metal alloys or complex oxide ceramics. The PBT perovskite phase structure was prepared by the conventional solid state reaction technique. The sample was analyzed by X-ray Diffraction (XRD), Particle size determination, SEM and EDX. The comparison of XRD results with JCPDS files confirmed the tetragonal structure of the sample with $\mathrm{a}=\mathrm{b} \neq \mathrm{c}$ and $\alpha=\beta=\gamma=90^{\circ}$. Scanning electron microscopy (SEM) studies revealed that its particle size was in the nanometer range. It also confirmed the calculated value of particle size from Debye Scherrer's formula. EDX spectrum shows the elements of the sample. X-ray instrumental peak broadening analysis was used to evaluate the size and lattice strain by the Williamson-Hall Plot method.
\end{abstract}

\section{Keywords}

Lead Barium Titanate (PBT), XRD, SEM, EDX, Debye Scherrer's Formula, Instrumental Broadening, Williamson-Hall Plot Method

\section{Introduction}

Ceramic materials are inorganic, non-metallic materials and things made from them. They may be crystalline or

${ }^{*}$ Corresponding author.

How to cite this paper: Vinila, V.S., Jacob, R., Mony, A., Nair, H.G., Issac, S., Rajan, S., Nair, A.S., Satheesh, D.J. and Isac, J. (2014) X-Ray Diffraction Analysis of Nano Crystalline Ceramic $\mathrm{PbBaTiO}_{3}$. Crystal Structure Theory and Applications, 3, 57-65. http://dx.doi.org/10.4236/csta.2014.33007 
partly crystalline. They are formed by the action of heat and subsequent cooling. They are brittle, hard, strong in compression, and weak in shearing and tension. They withstand chemical erosion that occurs in an acidic or caustic environment. Ceramics generally can withstand very high temperatures such as temperatures that range from $1000^{\circ} \mathrm{C}$ to $1600^{\circ} \mathrm{C}\left(1800^{\circ} \mathrm{F}\right.$ to $\left.3000^{\circ} \mathrm{F}\right)$. The Lead Barium Titanate (PBT) is a type of perovskite ceramic superconductor with high dielectric constant. Before final heating at $900^{\circ} \mathrm{C}$, the material PBT is treated at different temperatures, $30^{\circ} \mathrm{C}, 500^{\circ} \mathrm{C}$ and $850^{\circ} \mathrm{C}$. High dielectric constant (High-K) ceramic composites have become potential candidate materials for integration into high frequency electronics. Detailed understanding of this class of materials will help electronic industry in planning, design and processing of these materials.

In this work the authors describe the preparation of PBT ceramic material and it is characterized to show good quality, homogeneity and the desired stoichiometry of the sample prepared. The results were analyzed by X-ray diffraction (XRD), SEM, EDX. The particle size was determined from XRD details by Debye Scherrer formula. The SEM studies revealed that its particle size was in hundred nanometer range. The EDX spectrum of PBT gave the information on the elemental composition of the material. The particle size and strain of the material were calculated by Instrumental Broadening and Williamson-Hall Plot method.

\section{Materials and Experimental Methods}

PBT has perovskite structure. The perovskite structure is adopted by many oxides that have the chemical formula $\mathrm{ABO}_{3}[1]$. The representative structure of perovskite compounds is cubic, the compounds in this family may possess some distortion. The orthorhombic and tetragonal phases are most common variants. The sample with the chemical formula $\mathrm{PbBaTiO}_{3}$ were prepared by the conventional solid state reaction technique according to the molecular formula. For the prepared sample, the reagent grade chemicals of high purity (99.99\%) Lead Oxide, Barium Carbonate and Titanium Oxide powders were used as the raw materials and weighed according to their molecular formula.

The powders of the required ceramics were mixed mechanically. Mechanical mixing is usually done by hand mixing in agate mortar for very long time. Then ball milled with suitable balls to insure homogeneity and milling. Then the material calcined at different temperatures, $30^{\circ} \mathrm{C}, 500^{\circ} \mathrm{C}, 850^{\circ} \mathrm{C}$ and $900^{\circ} \mathrm{C}$. After the furnace is off, on cooling the oxygen is allowed to flow into the furnace at intervals (Oxygen Annealing). A final furnace temperature of $900^{\circ} \mathrm{C}$ is maintained for the intermediate firings. A temperature much higher than this will result in a material that is much harder to regrind. Temperatures above $1030^{\circ} \mathrm{C}$ may destroy the crystal structure. Then $\mathrm{X}$-ray diffraction spectrum of these materials was taken. For experimental conformation of the calculated particle size value, Scanning Electron Microscopy (SEM) photograph was taken. From EDX, the composition details of the prepared ceramics were determined (Figure 1).

The red spheres are $\mathrm{X}$ atoms (usually oxygens), the blue spheres are B-atoms (a smaller metal cation, such as $\mathrm{Ti}$ ), and the green spheres are the A-atoms (a larger metal cation, such as $\mathrm{Pb} / \mathrm{Ba}$ ). Pictured is the undistorted cubicstructure; the symmetry is lowered to orthorhombic, tetragonal or trigonal in many perovskites [2].

\subsection{XRD Analysis}

X-ray Diffraction pattern for the four different temperatures in steps for the sample PBT (Figure 2) was taken using Bruker AXS D8 advance diffractometer. The diffractometer with radiations of wavelength $1.54184 \AA$

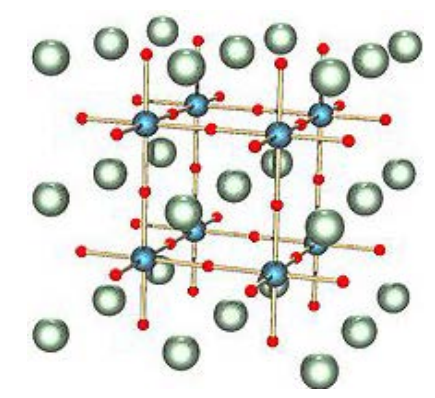

Figure 1. Structure of a perovskite with a chemical formula $\mathrm{ABX}_{3}$. 

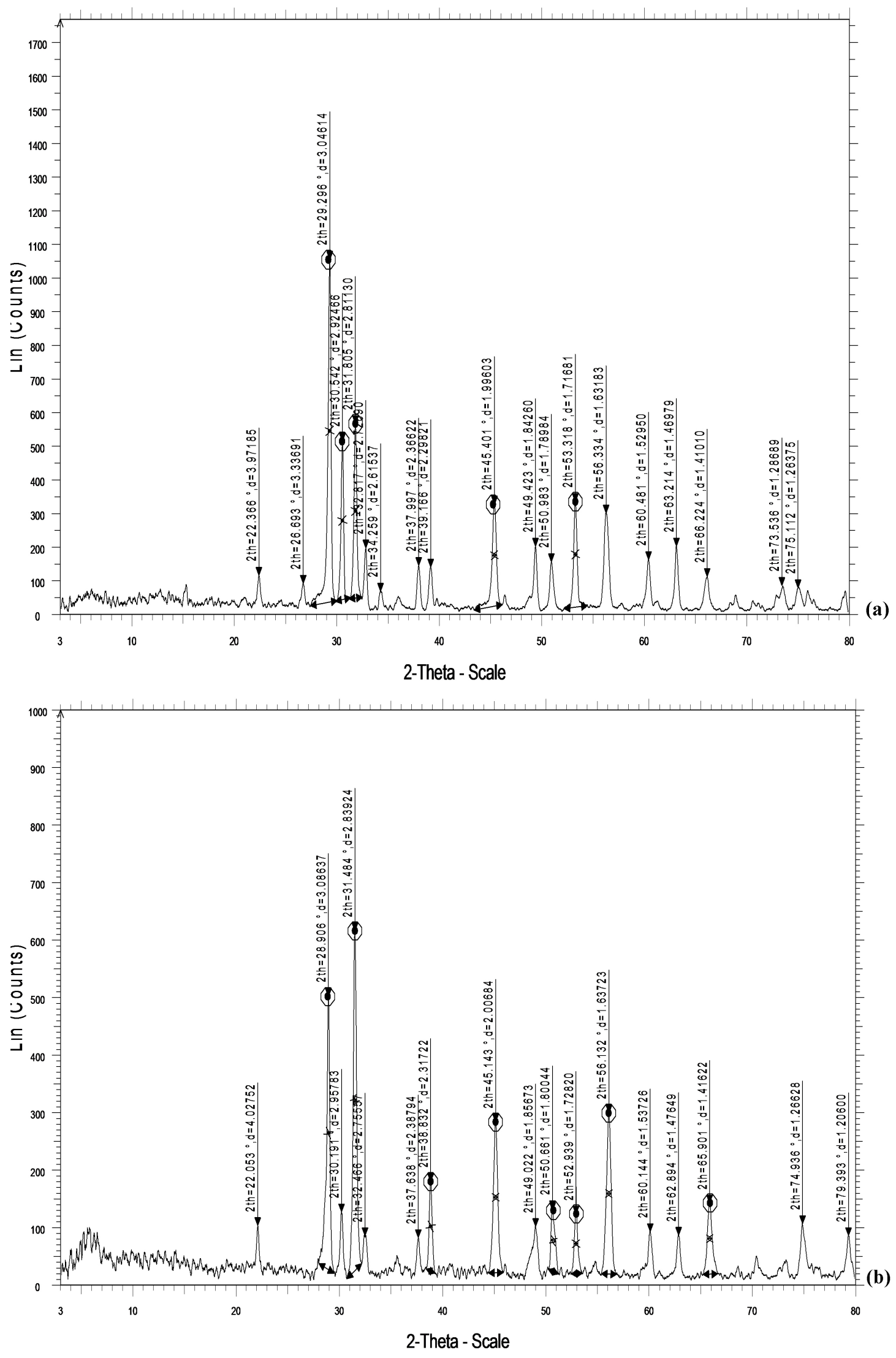

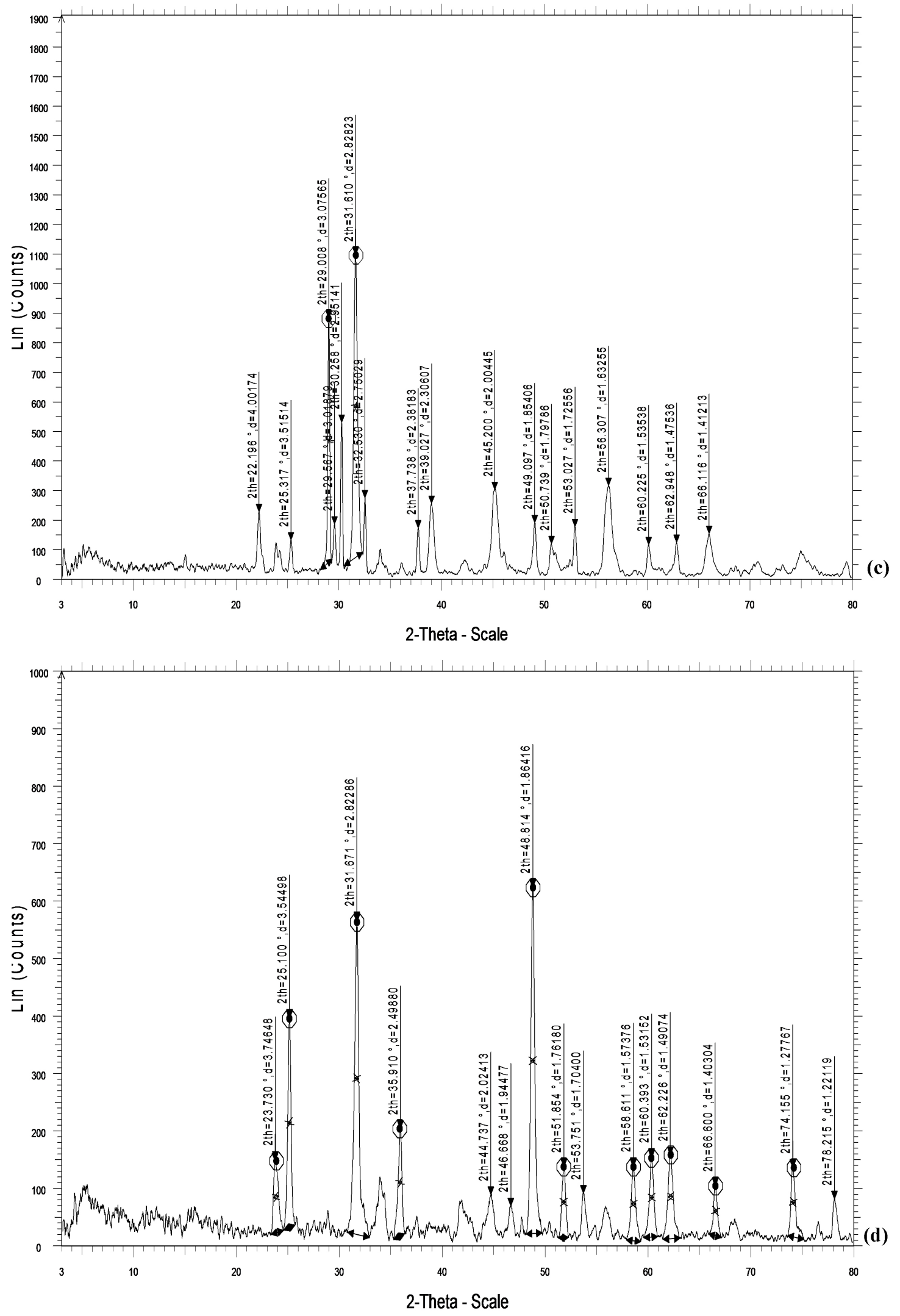

Figure 2. XRD of PBT at temperatures (a) $30^{\circ} \mathrm{C}$; (b) $500^{\circ} \mathrm{C}$; (c) $850^{\circ} \mathrm{C}$ and (d) $900^{\circ} \mathrm{C}$. 
having Nickel filter, equipped with X-ray generator 1140/90/96 having X-ray source KRISTALLOFLXE 780 KF, 4KE with wide angle goniometer PW1710/70 with single pen recorder pm 8203 and channel control PW1390 at $35 \mathrm{kV}, 10 \mathrm{~mA}$ is used for the purpose. The scanning speed of the specimen is 2 degree/minute. From the XRD results, the obtained d values compared with the JCPDS (Joint Committee on Powder Diffraction Standards) file values. So that it can be concluded that this crystal is found to be tetragonal system:

$$
a=b=3.761, c=11.942, \alpha=\beta=\gamma=90^{\circ}
$$

From the XRD data, we can calculate the particle size of PBT by using Debye Scherrer equation. Here the authors studied the change of XRD spectrum of PBT at different treating temperatures.

\subsection{Particle Size Measurements}

Crystal structures are solved by analyzing the intensities of diffracted X-ray beams. X-ray diffraction profile may be used to measure the average crystal size in the sample provided the average diameter is less than $200 \AA$. The lines in a powder diffraction pattern are of finite breadth but if the particles are very small, the lines are broaden than usual. The broadening decreases with the increase in particle size. The particle size for PBT was calculated from X-ray diffraction profiles of strong reflections with intensity \% by measuring the full width at half maximum (FWHM). The Debye Scherrer equation for calculating the particle size is given by [3].

$$
D=\frac{K \lambda}{\beta \cos \theta}
$$

where $K$ is the Scherrer constant, $\lambda$ is the wavelength of light used for the diffraction, $\beta$ is the "full width at half maximum" of the sharp peaks and $\theta$ is the angle measured. The Scherrer constant $(K)$ in the above formula accounts for the shape of the particle and is generally taken to have the value 0.9. The results reveal that the particle size is less than $100 \mathrm{~nm}$. The particle size of PBT at different temperatures is shown in Table 1.

\subsection{XRD-Instrumental Broadening}

When particle size is less than $100 \mathrm{~nm}$, appreciable broadening in X-ray diffraction lines will occur. Diffraction pattern will show broadening because of particle size and strain. The observed line broadening will be used to estimate the average size of the particles. The total broadening of the diffraction peak is due to sample and the instrument. The sample broadening is described by

$$
F W(s) \times \cos \theta=\frac{K \times \lambda}{\text { size }}+4 \times \text { strain } \times \sin \theta
$$

The total broadening $\beta_{t}$ equation is described by

$$
\beta_{t}^{2} \approx\left\{\frac{0.9 \lambda}{D \cos \theta}\right\}^{2}+\{4 \varepsilon \tan \theta\}^{2}+\beta_{0}^{2}
$$

where $D$ is average particle size, $\varepsilon$ is strain and $\beta_{0}$ is instrumental broadening. Instrumental broadening is presented in Figure 3.

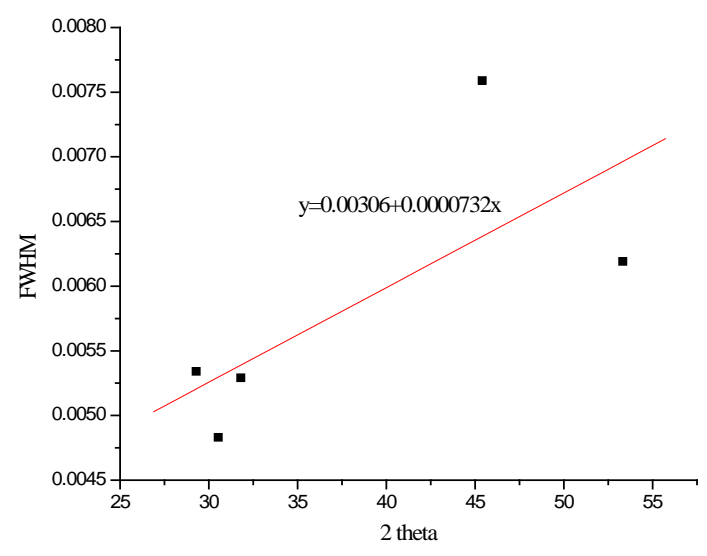

Figure 3. Typical instrumental broadening. 
Table 1. The particle size of PBT at different temperatures, calculated by Debye Scherrer formula.

\begin{tabular}{cccc}
\hline Data of high intensity peak of XRD at different temperatures & $\Theta$ (degree) & B (radian) $\left(\times 10^{-3}\right)$ & Particle size $(\mathrm{nm})$ \\
\hline $\mathrm{PBT}-30^{\circ} \mathrm{C}$ & 14.646 & 7.10 & 19.55 \\
$\mathrm{PBT}-500^{\circ} \mathrm{C}$ & 15.741 & 6.94 & 20.00 \\
$\mathrm{PBT}-850^{\circ} \mathrm{C}$ & 15.808 & 6.04 & 22.97 \\
$\mathrm{PBT}^{\circ}-900^{\circ} \mathrm{C}$ & 24.409 & 5.34 & 25.99 \\
\hline
\end{tabular}

\subsection{Williamson-Hall Plot}

This method is attributed to G. K. Williamson and his student, W. H. Hall. It relies on the principle that the approximate formulae for size broadening, $\beta_{L}$, and strain broadening, $\beta_{e}$, vary quite differently with respect to Bragg angle, $\theta$. Williamson and Hall proposed a method for deconvoluting size and strain broadening by looking at the peak width as a function of $2 \theta$ [4].

$$
\begin{aligned}
& \beta_{L}=\frac{K \lambda}{L \cos \theta} \\
& \beta_{e}=C \varepsilon \tan \theta
\end{aligned}
$$

One contribution varies as $1 / \cos \theta$ and the other as $\tan \theta$. If both contributions are present then their combined effect should be determined by convolution. The simplification of Williamson and Hall is to assume the convolution is either a simple sum or sum of squares. Using the former equations, we get:

$$
\beta_{\text {tot }}=\beta_{e}+\beta_{L}=C \varepsilon \tan \theta+\frac{K \lambda}{L \cos \theta}
$$

If we multiply this equation by $\cos \theta$ we get:

$$
\beta_{\text {tot }} \cos \theta=C \varepsilon \sin \theta+\frac{K \lambda}{L}
$$

and comparing this to the standard equation for a straight line ( $m=$ slope; $c=$ intercept) $y=m x+c$.

We see that by plotting $\beta_{t o t} \cos \theta$ versus $\sin \theta$ we obtain the strain component from the slope $(C \varepsilon)$ and the size component from the intercept $(K \lambda / L)$. Such a plot is known as a Williamson-Hall Plot. However the WilliamsonHall method has many assumptions: its absolute values should not be taken too seriously but it can be a useful method if used in the relative sense; for example a study of many powder patterns of the same chemical compound, but synthesized under different conditions, might reveal trends in the crystallite size/strain which in turn can be related to the properties of the product. Figure 4 shows Williamson Hall Plot.

\subsection{SEM Analysis}

Morphology has been analyzed from JEOL Model JSM-6390LV Scanning Electron Microscope (SEM). Scanning electron microscopy (SEM) is a method for high resolution surface imaging. The SEM uses an electron beam for surface imaging. The advantages of SEM over light microscopy are greater magnification and much larger depth of field. Different elements and surface topographies emit different quantity of electrons, due to which the contrast in a SEM micrograph (picture) is representative of the surface topography and distribution of elemental composition on the surface. In SEM analyses the surface of solid objects, producing images of higher resolution than optical microscopy. It produces representations of three dimensional samples from a diverse range of materials. Figure 5 is the surface morphology of PBT. The particle size measurement through SEM reveals its maximum dimensions always less than $100 \mathrm{~nm}$.

\subsection{Energy Dispersed X-Ray Spectrograph (EDX)}

EDX (Figure 6) shows the composition details of the prepared ceramic powders. The instrument used for this measurement is ISIS Link Oxford Instrument UK. This technique generally associated with Scanning Electron Microscope (SEM). In this technique an electron beam of $10-20 \mathrm{KeV}$ strikes the surface of a sample which causes X-ray to be emitted from point of incidence. The energy of the X-ray emitted depends on material under examination. The energy of the characteristic X-ray emitted from the different elements is different and thus it gives the unavoidable signature of the particular element. When an X-ray strikes the detector, it will generate a photoelectron which in turn generates electron-hole pairs. A strong electric field attracts the electrons and holes 


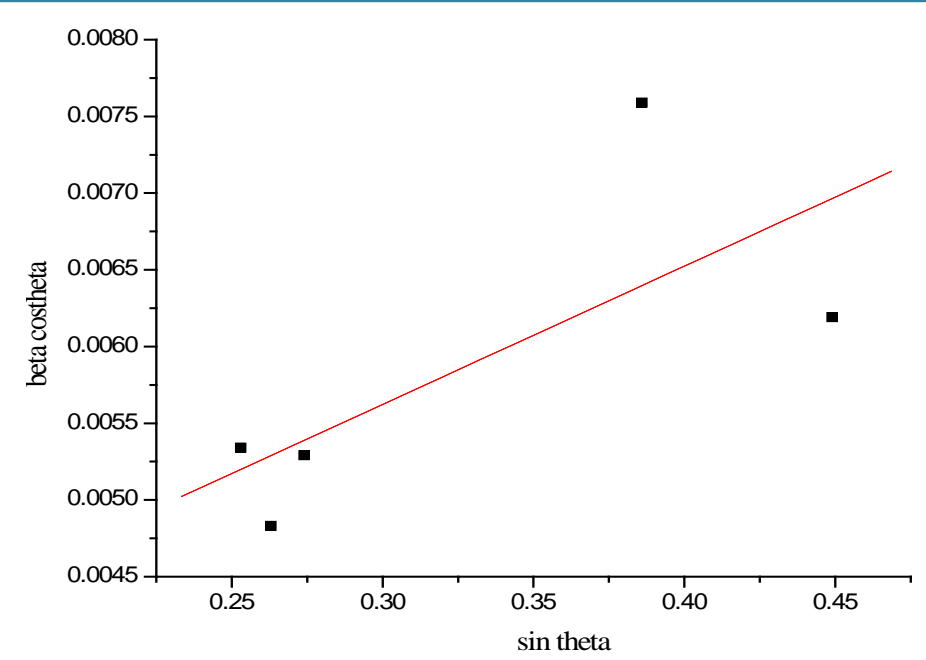

Figure 4. Williamson hall plot. Slope $=0.00901$; Y intercept $=0.00495$; Correlation coefficient $=0.72404$; Standard deviation of the fit $=0.000868$.

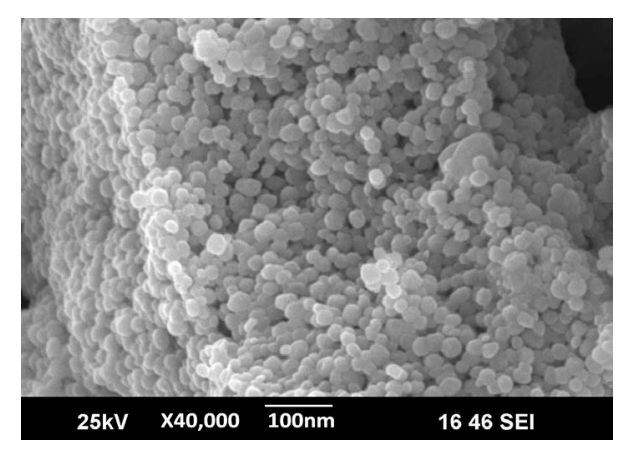

Figure 5. SEM photograph of PBT.

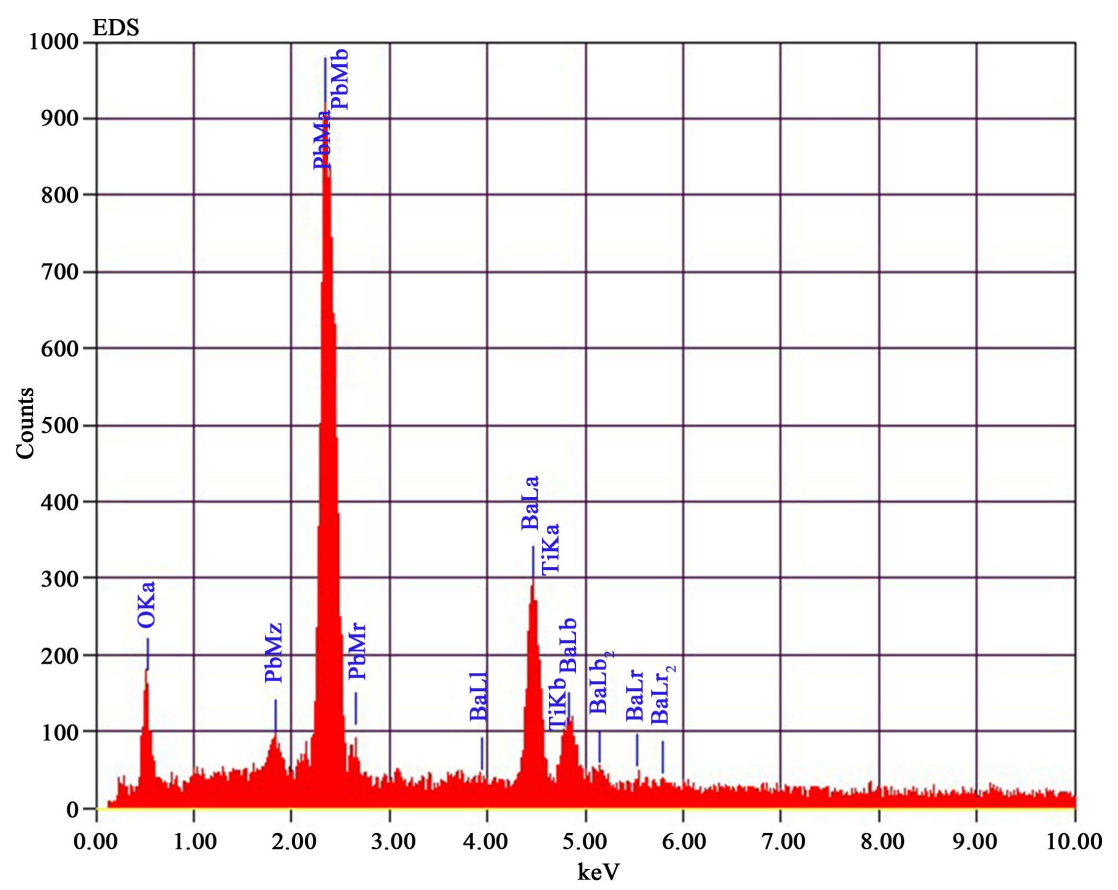

Figure 6. EDX of PBT. 
towards the opposite ends of the detector. The size of the pulse thus generated depends on the number electron-hole pairs created, which in turn depends on the energy of the incoming X-ray. In this method however elements with low atomic number are difficult to be detected. The detector which is lithium doped silicon (SiLi) is protected by a beryllium window and operated at liquid nitrogen temperatures. The absorption of the soft $\mathrm{X}$-rays by the beryllium decreases the sensitivity below an atomic number of 11 .

\section{Results and Discussion}

The XRD patterns of PBT powder obtained for various annealing temperatures are shown in Figure 2. XRD spectrum for the different temperatures gave a clear idea about the maximum intensity peak shifting corresponds to the different treating temperatures. And also get the maximum intensity peak difference. The temperature increases, the highest peaks in the XRD spectrums shifts from left to right through the $2 \theta$ axis and the highest intensity peak decreases.

The atoms undergo thermal vibration about their mean positions even at the absolute zero of temperature, and the amplitude of this vibration increases as the temperature increases. Increased thermal vibration of the atoms, as the result of an increase in temperature, the unit cell expands, causing changes in plane spacing $d$ and therefore in the $2 \theta$ positions of the diffraction lines. And also the intensities of the diffraction lines decrease.

When a material heated from room temperature to high temperature, the amplitude of the thermal vibrations increased. The amplitude is very higher than that at room temperature. After heating, the material cooled to room temperature. The amplitude of the atomic vibrations of the material was decreased. But it couldn't arrive at the initial amplitude. The atomic vibration amplitude of the heated material was higher than initial amplitude. That means, the atomic vibration amplitude increases, the intensity of the diffracted beam also decreases because it has the effect of smearing out lattice planes. Thus the reinforcement of waves scattered at the Bragg angle by various parallel planes is not as perfect as it is for a crystal with fixed atoms. This reinforcement requires that the path difference, which is a function of the plane spacing $d$, between waves scattered by adjacent planes be an integral number of wavelengths. The thickness of the planes is $2 u$, where $u$ is the average displacement of an atom from its mean position. Under these conditions reinforcement is no longer perfect, and it becomes more imperfect as the ratio $\mathrm{u} / \mathrm{d}$ increases, i.e., as the temperature increases since that increases $\mathrm{u}$, or as $\theta$ increases, since high- $\theta$ reflections involve planes of low $\mathrm{d}$ value. Thus the intensity of a diffracted beam decreases as the temperature is raised. In intensity calculations, the temperature factor $\mathrm{e}^{-2 \mathrm{M}}$ decreases as $2 \theta$ increases [5].

From Figure 2, the peak broadening in the XRD patterns clearly indicated the nature of the very small nanocrystals. From the width of the XRD peak, the mean crystalline size can be calculated using Debye Scherrer's equation. From Table 1, particle size of the material PBT increases with respect to the treating temperature increasing. And also it is realized that, $\theta$ increases with temperature. The results revealed that the particle size is less than $100 \mathrm{~nm}$. The diffraction data revealed that the material belongs to tetragonal symmetry. The results were compared with JCPDS data along with XPERT-PRO programme.

Heat treatment causes the particles to anneal and form larger grains, which of course indicates that the particles become larger. Hence, the large particle size of sample at $900^{\circ} \mathrm{C}$ is expected. This also agrees with the higher crystallinity, as having larger grains means more long-range order, and hence more crystallinity [6] [7].

The size and strain of the experimentally observed broadening of several peaks are computed simultaneously using least squares method (Figure 3). When, particle size becomes smaller, due to size effect, the peaks become broad and widths larger. The broadening of peak may also occur due to micro strains of the crystal structure arising from defects like dislocation and twinning [8].

Here, Williamson-Hall plot is plotted with $\sin \theta$ on the $\mathrm{x}$-axis and $\beta \cos \theta$ on the $\mathrm{y}$-axis (in radians). A linear fit is got for the data. From this fit, particle size and strain are extracted from y-intercept and slope respectively. The extracted particle size is $26.04 \mathrm{~nm}$ and strain is 0.00901 from Williamson Hall Plot (Figure 4).

Figure 5 shows SEM image of PBT. The SEM photograph revealed maximum dimensions of the particles to be always less than $100 \mathrm{~nm}$. This is an experimental proof of the theoretical calculation of particle size by Debye Scherrer equation from XRD data.

EDX (Figure 6) spectrum of PBT gave the information on the elemental composition of the material. The elemental compositions agree with the stoichiometric relations of the prepared compound.

The EDX spectrums obtained give the material under investigation.

From the EDX spectrum, the four dominant peak positions at $2.342 \mathrm{keV}, 4.465 \mathrm{keV}, 2.307 \mathrm{keV}, 0.525 \mathrm{keV}$ correspond quite well to the energy pattern of the corresponding materials $(\mathrm{Pb}, \mathrm{Ba}, \mathrm{Ti}$, and $\mathrm{O}$ ) reported in the EDAX international chart, giving the evidence that $\mathrm{Pb}$ and $\mathrm{Ba}$ are dominant in PBT samples. Table 2 shows the 
Table 2. Material Content (EDX).

\begin{tabular}{cc}
\hline Material & Content (\%) \\
\hline 70.07 & $\mathrm{~Pb}$ \\
24.53 & $\mathrm{Ba}$ \\
3.35 & $\mathrm{Ti}$ \\
2.05 & $\mathrm{O}$ \\
\hline
\end{tabular}

percentage of the elements in the prepared PBT sample.

\section{Conclusion}

In this work, PBT ceramics were prepared successfully by the conventional solid state reaction technique and studied by XRD, SEM, EDX, and particle size measurement. XRD data confirmed the formation of the perovskite phase structure and the average particle size. The XRD spectrums of the PBT at different temperatures indicate that, according to increasing treating temperature, the $\theta$ value and particle size also increases. From SEM analysis, the morphology images show the approximate size of the nano particles. The value of particle size calculated from the Williamson-Hall plot method is in agreement with that of the particle size measured from Debye Scherrer formula. The EDX analysis indicates that the elements exist in the sample and it agrees with the stoichiometric relations of the prepared compound.

\section{Acknowledgements}

The authors are thankful to Kerala State Council for Science, Technology And Environment (KSCSTE), Thiruvanathapuram for granting the financial assistance, SAIF, Cochin for providing the data analysis and to the Principal, CMS College, Kottayam, Kerala for providing the facilities.

\section{References}

[1] Galasso, F.S. (1969) Structure, Properties and Preparation of Perovskite Type Compounds. Pergamon Press, Oxford.

[2] Navrotsky, A. (1998) Energetics and Crystal Chemical Systematics among Ilmenite, Lithium Niobate, and Perovskite Structures. Chemistry of Materials, 10, 2787-2793. http://dx.doi.org/10.1021/cm9801901

[3] West, A.R. (1974) Solid State Chemistry and It’s Applications. Wiley, New York.

[4] Williamson, G.K. and Hall, W.H. (1953) X-Ray Line Broa Dening from Filed Aluminium and Wolfram. Acta Metallurgica, 1, 22-31. http://dx.doi.org/10.1016/0001-6160(53)90006-6

[5] Cullity, B.D. (1978) Elements of X-Ray Diffraction. 2nd Edition, Addison-Wesley Publishing Company Inc., Phillippines.

[6] Xie, Q. and McCourt, F. (2008) Nanotechnology Engineering NE 320L Lab Manual. University of Waterloo, Waterloo.

[7] So, W.W., Jang, J.S., Rhee, Y.W., Kim, K.J. and Moon, S.J. (2001) Preparation of Nanosized Crystalline CdS Particles by the Hydrothermal Treatment. Journal of Colloid and Interface Science, 237, 136-141. http://dx.doi.org/10.1006/jcis.2001.7489

[8] Ghosh, S.C., Thanachayanont, C. and Dutta, J. (2004) Studies on Zinc Sulphide Nanoparticles for Field Emission Devices. The 1st ECTI Annual Conference (ECTI-CON2004), Pattaya, 13-14 May 2004. 
Scientific Research Publishing (SCIRP) is one of the largest Open Access journal publishers. It is currently publishing more than 200 open access, online, peer-reviewed journals covering a wide range of academic disciplines. SCIRP serves the worldwide academic communities and contributes to the progress and application of science with its publication.

Other selected journals from SCIRP are listed as below. Submit your manuscript to us via either submit@scirp.org or Online Submission Portal.
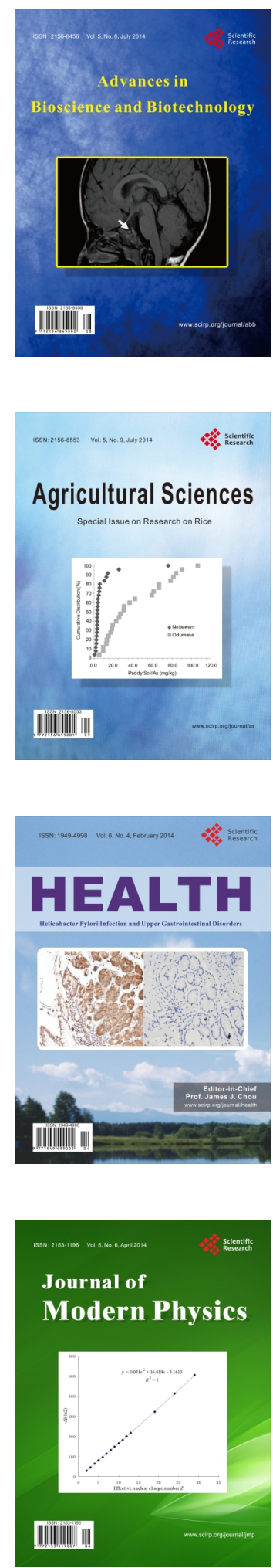
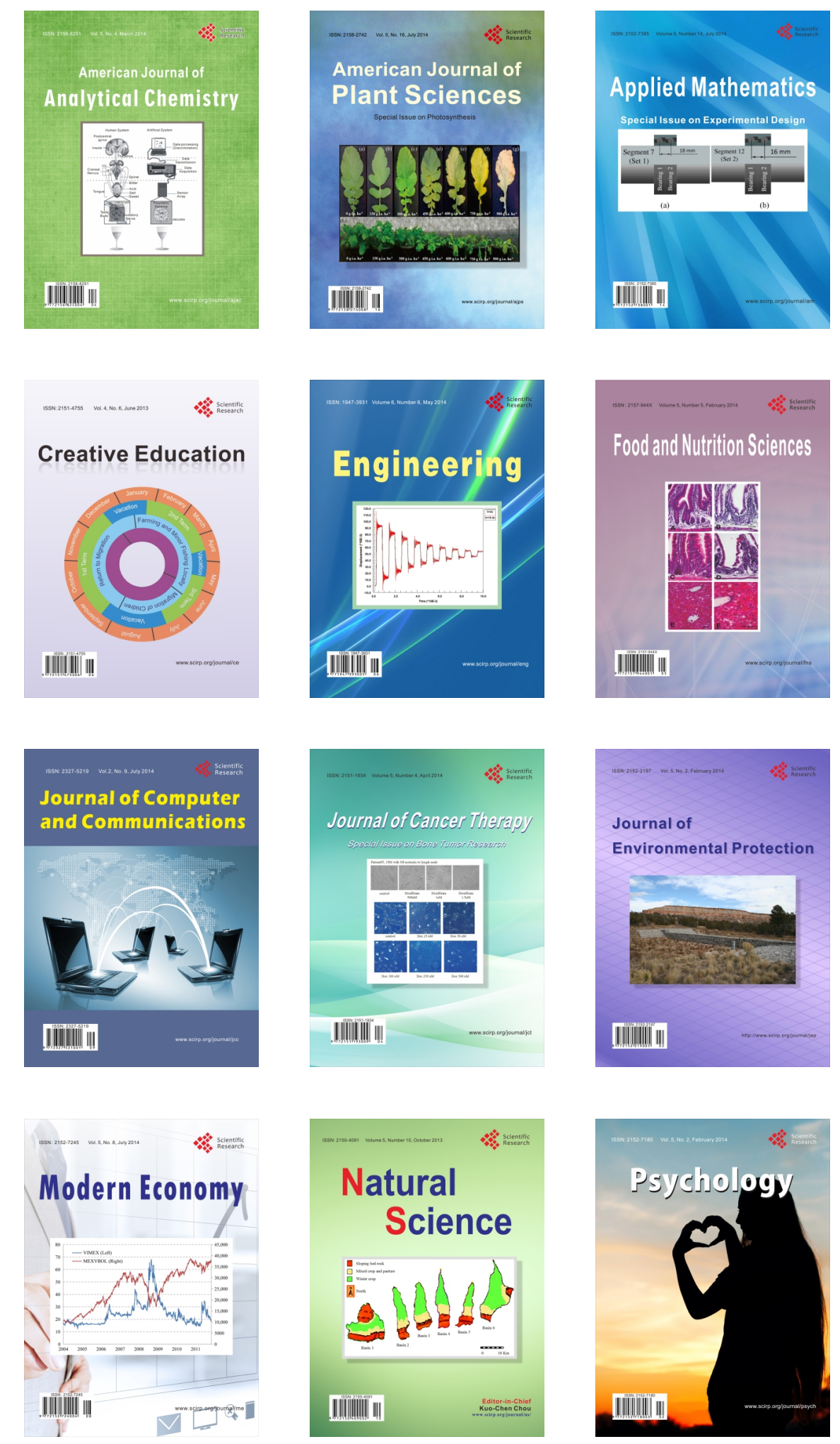